\title{
Efficacy and Tolerability of Lacosamide in the Concomitant Treatment of 130 Patients Under 16 Years of Age with Refractory Epilepsy A Prospective, Open-Label, Observational, Multicenter Study
in Spain
}

Carlos Casas-Fernández, ${ }^{1}$ Antonio Martínez-Bermejo, ${ }^{2}$ Miguel Rufo-Campos, ${ }^{3}$ Patricia Smeyers-Durá, ${ }^{4}$ José L. Herranz-Fernández, ${ }^{5}$ Salvador Ibánez-Micó, ${ }^{1}$ Jaume Campistol-Plana, ${ }^{6}$ Helena Alarcón-Martínez ${ }^{1}$ and Jaime Campos-Castello ${ }^{7}$

1 Neuropediatric Department, Hospital Universitario Virgen de la Arrixaca, Murcia, Spain

2 Neuropediatric Department, Hospital Universitario La Paz, Madrid, Spain

3 Neuropediatric Department, Instituto Hispalense de Pediatría, Seville, Spain

4 Neuropediatric Department, Hospital Universitario La Fe, Valencia, Spain

5 Neuropediatric Department, Hospital Universitario Marqués de Valdecilla, Universidad de Cantabria, Santander, Spain

6 Neuropediatric Department, Hospital Universitario San Juan de Dios, Barcelona, Spain

7 Neuropediatric Department, Hospital Clínico San Carlos, Madrid, Spain

\section{Abstract}

Background: The safety and effectiveness of lacosamide, an antiepileptic drug (AED) that selectively enhances the slow inactivation of voltage-gated sodium channels without affecting rapid inactivation, has been demonstrated in randomized, double-blind, placebo-controlled trials in adults with focal epileptic seizures. Although lacosamide is approved for use in patients over 16 years of age, limited clinical experience exists for younger patients.

Objective: To assess the efficacy and tolerability of lacosamide in children with refractory epilepsy.

Design/Methods: The trial was a prospective, open-label, observational, multicenter study. A total of 130 patients aged less than 16 years (range 6 months to 16 years) with refractory epilepsy who had initiated treatment with lacosamide were enrolled at 18 neuropediatric units in hospitals across Spain. Patients with a variety of etiologies were enrolled, including those with partial epilepsies and symptomatic, generalized epilepsy syndromes. Lacosamide (VIMPAT $^{\circledR}$; UCB Pharma SA, Brussels, Belgium) was primarily administered once every 12 hours as an oral solution or as an oral tablet, with an initial dose of $1-2 \mathrm{mg} / \mathrm{kg} / \mathrm{day}$ in the majority of cases. The majority of patients were also receiving stable concomitant therapy with $\geq 1$ other AED. Treatment response 
to lacosamide was determined by assessing the change in seizure frequency after 3 months of lacosamide therapy. Responders were defined as patients who achieved a seizure frequency reduction of $>50 \%$. Tolerability was assessed by the reporting of adverse effects, laboratory testing, and electroencephalography recordings.

Results: Lacosamide was dosed at a mean of $6.80 \pm 2.39 \mathrm{mg} / \mathrm{kg} / \mathrm{day}$. After 3 months of lacosamide therapy, $62.3 \%$ of patients achieved a $>50 \%$ reduction in seizure frequency, with complete seizure suppression being reported in $13.8 \%$ of patients. Adverse effects occurred in 39 patients (30\%), but no doseresponse relationship was observed in terms of these events. In ten patients, instability, difficulty walking, an inability to relate to subjective elements, and blurred vision or dizziness were reported. A total of 13 patients discontinued treatment - in five of these patients, symptom intensity remained unchanged despite dose reduction, which led to treatment discontinuation. The symptoms were markedly different in each patient, preventing determination of a causal factor(s).

Conclusions: The results of this study provide preliminary evidence for the efficacy of lacosamide in children with refractory epilepsy. Further evaluation in a randomized, controlled trial is needed to validate the efficacy in this population and to fully investigate the adverse effects described here. We recommend an initial dose of $1-2 \mathrm{mg} / \mathrm{kg} / \mathrm{day}$, uptitrated to $6-9 \mathrm{mg} / \mathrm{kg} / \mathrm{day}$ over 4-6 weeks.

\section{Introduction}

The antiepileptic drug (AED) lacosamide is chemically composed of acetamido-N-benzyl-3methoxypropionamide, an amino acid with a molecular weight of $250.3 \mathrm{~g} / \mathrm{mol}$, and is highly soluble in water $(25 \mathrm{mg} / \mathrm{mL}){ }^{[1-4]}$ The mechanism of action through which lacosamide exerts its antiepileptic effect is unique in that it selectively enhances slow inactivation of voltage-gated sodium channels without affecting rapid inactivation. ${ }^{[1-4]}$ This reduces the long-term availability of these sodium channels, which results in diminished pathological hyper-excitability without compromising physiological activity. ${ }^{[1-4]}$ Therefore, lacosamide does not completely block voltage-gated sodium channels but, rather, acts as a modulator of these channels. ${ }^{[1-4]}$

With regard to pharmacokinetics, lacosamide has oral bioavailability of approximately $100 \%$ and a very low plasma protein binding rate $(<15 \%)$;
$95 \%$ is excreted in urine, $40 \%$ as unaltered lacosamide and $30 \%$ as inactive O-desmethyl metabolite. ${ }^{[2-6]}$ The maximum plasma drug concentration $\left(\mathrm{C}_{\max }\right)$ is reached between 1 and 2 hours following oral administration, with an elimination half-life $\left(t_{1 / 2}\right)$ of 13 hours, thereby enabling administration of two doses per day. ${ }^{[2-6]}$ No pharmacokinetic interactions have been observed in various clinical trials with other AEDs, digoxin, metformin, omeprazole, or oral contraceptives containing ethinylestradiol and levonorgestrel. ${ }^{[2-6]}$

The effectiveness and safety of lacosamide have been demonstrated in three randomized, doubleblind, placebo-controlled clinical trials conducted in adult patients with focal epileptic seizures. Although lacosamide is approved for use in patients over 16 years of age, ${ }^{[6-8]}$ limited clinical experience exists for younger patients. ${ }^{[9,10]}$ Therefore, our study was conducted to evaluate the efficacy and tolerability of lacosamide in children aged less than 16 years with refractory epilepsy. 


\section{Methods}

\section{Study Design}

This was a prospective, open-label, observational, multicenter study conducted at 18 neuropediatric units across Spain (listed in the Appendix). Patients were recruited by neuropediatric doctors at each participating unit over a period of 12 months, and were eligible for the study if they had already initiated treatment with lacosamide after a lack of response to prior antiepileptic treatment, defined as a minimum of 2 months without a clinical response to previously administered AEDs. Lacosamide had been prescribed because the neuropediatric doctor believed the patient could benefit from its use. Patients were required to have previously been treated with at least two AEDs, to be less than 16 years of age, and to have a diagnosis of refractory epilepsy that met the current International League Against Epilepsy (ILAE) criteria for refractory or difficult-to-control epilepsy. ${ }^{[11]}$ Patients with any neurodegenerative diseases were excluded. Written informed consent was obtained from the parents of children under 16 years of age, conforming to the recommendations of the Declaration of Helsinki. The informed consent document stated that the Summary of Product Characteristics for lacosamide clearly indicates the use of the drug from the age of 16 years and highlighted the potential side effects that should be monitored with special attention. The manufacturer of lacosamide (UCB Pharma) had no involvement in the study.

Lacosamide (VIMPAT ${ }^{\circledR}$; UCB Pharma SA, Brussels, Belgium) was primarily used as an oral solution $(15 \mathrm{mg} / 1 \mathrm{cc})$ or tablets $(50 \mathrm{mg}, 100 \mathrm{mg}$, $150 \mathrm{mg}$, and $200 \mathrm{mg}$ ), administered once every 12 hours. The initial dose ranged from 1 to $2 \mathrm{mg} / \mathrm{kg}$ /day in the majority of cases $(89.2 \%)$. Patients were uptitrated from 1 or $2 \mathrm{mg} / \mathrm{kg} /$ day to 6-9 mg/kg/day over 4-6 weeks. Lacosamide was acquired by the patients from pharmacies through the Spanish National Health prescription service. Concomitant AEDs (co-AEDs) were maintained at a stable dose during the study. Treatment did not exceed 6 months if there was an increase in seizure frequency, if the onset of adverse effects resulted in treatment withdrawal, or if the clinical situation did not improve and the medication was discontinued. Two-thirds of patients $(66 \%)$ had been on treatment for 6 months or more when the data were collected. In cases where co-AEDs was used, they were the same drugs the patients had been taking prior to initiation of lacosamide.

\section{Evaluations and Outcome Measures}

Before lacosamide treatment was started, the clinical status of patients was monitored by the participating neuropediatric doctors every 6 months, with laboratory and electroencephalography (EEG) assessments being conducted if deemed clinically necessary. Patients were then followed up and monitored by these participating doctors according to a protocol established by general consensus at the start of the study, with clinical and laboratory assessments completed quarterly.

Response to treatment was evaluated by the difference between the number of epileptic seizures occurring during lacosamide treatment and the number of epileptic seizures occurring in the period prior to starting treatment with lacosamide. The number of seizures was provided by the patients' parents, who completed a 'seizure calendar'. The seizure calendar was delivered to parents at the start of treatment with lacosamide, and thereafter they would fill it in. Prior to starting lacosamide treatment, some (but not all) patients had been creating and filling in their own seizure calendar. After the start of lacosamide treatment, however, all of them filled in this calendar.

Seizure frequency was measured during the 3-month period prior to lacosamide therapy and after 3 months of lacosamide therapy. The main efficacy outcome measure was the proportion of responders to lacosamide therapy after 3 months of treatment, which was defined as a reduction in seizure frequency of $>50 \%$. Complete control was defined as no seizures occurring in the analyzed period. Patients were divided into five categories according to the level of their response to treatment: complete seizure control (group A); a reduction in seizure frequency of $>75 \%$ (group B); a reduction in seizure frequency of $>50 \%$ to $75 \%$ (group C); no change in seizure frequency 
Table I. Characteristics of patients enrolled in the study $(N=130)$

\begin{tabular}{|c|c|}
\hline Characteristic & Value \\
\hline Male sex (n [\%]) & $72[55.4]$ \\
\hline \multicolumn{2}{|l|}{ Age (years) } \\
\hline Mean \pm standard deviation & $8.01 \pm 4.25$ \\
\hline Range & $0.5-16$ \\
\hline \multicolumn{2}{|l|}{ Etiology (n [\%]) } \\
\hline Symptomatic origin & $82[63.1]$ \\
\hline Presumed symptomatic origin & 36 [27.7] \\
\hline Idiopathic origin & $12[9.2]$ \\
\hline$>20$ seizures per month (n [\%]) & $70[53.8]$ \\
\hline \multicolumn{2}{|l|}{ Type of focal seizure (n [\%]) } \\
\hline Simple & $32[24.6]$ \\
\hline Complex & $93[71.5]$ \\
\hline Secondarily generalized & 84 [64.6] \\
\hline \multicolumn{2}{|l|}{ Use of co-AEDs $(n[\%])^{a}$} \\
\hline 1 co-AED & $41[31.5]$ \\
\hline 2 co-AEDs & 68 [52.3] \\
\hline 3 co-AEDs & $17[13.1]$ \\
\hline$>3$ co-AEDs & $3[2.3]$ \\
\hline \multicolumn{2}{|l|}{ Type of co-AED used ( $[\%]$ ) } \\
\hline Valproate & $59[45.4]$ \\
\hline Levetiracetam & 51 [39.2] \\
\hline Zonisamide & $23[17.7]$ \\
\hline Oxcarbazepine & 18 [13.8] \\
\hline Clobazam & 18 [13.8] \\
\hline Topiramate & $17[13.1]$ \\
\hline \multicolumn{2}{|c|}{$\begin{array}{l}\text { One patient reverted to monotherapy after a trial of combination } \\
\text { therapy and is therefore not included in this list. }\end{array}$} \\
\hline co-AED = concomitant antiepilept & \\
\hline
\end{tabular}

(group D); or an increase in seizure frequency (group E).

Tolerability was assessed by the recording of adverse effects and the attitudes adopted toward transient initial symptoms, a reduction in the dose of lacosamide or other AEDs, and lacosamide withdrawal. Usually the parents/family of the patient reported adverse effects unless the patient was capable of providing this information him- or herself, in which case reporting of adverse effects was done by the patient and their parents/family. Conventional laboratory tests (complete blood count, transaminasemia, amylasemia, blood glucose, creatininemia, cholesterolemia, and triglyceridemia) and EEG recordings were also performed.

\section{Statistical Analysis}

The analysis of the mean lacosamide dosage (in $\mathrm{mg} / \mathrm{kg} / \mathrm{day}$ ) according to the percentage control of seizures (level of response) was performed using the Kruskal-Wallis test. The association of AEDs with different levels of response was analyzed by the $\chi^{2}$ test. The analysis of the mean lacosamide dosage (in $\mathrm{mg} / \mathrm{kg} /$ day) in patients with and without adverse effects was performed using the Mann-Whitney test.

\section{Results}

Clinical Characteristics and Disposition of Subjects

Data on patient demographics and clinical characteristics are summarized in table I. Overall, 130 cases of refractory epilepsy were analyzed in patients under 16 years of age (mean age $8.01 \pm 4.25$ years; range 6 months to 16 years). Epilepsies of a symptomatic origin were due to perinatal pathology $(25.9 \%)$, malformations of cortical development [MCD] (19.7\%), other cerebral malformations (14.8\%), neuroectodermal disorders $(12.3 \%)$, central nervous system infections $(8.6 \%)$, metabolic diseases $(6.1 \%)$, genetic alterations $(4.9 \%)$, mesial sclerosis $(3.7 \%)$, cerebrovascular disease $(2.4 \%)$, and presumed auto-

Table II. Concomitant antiepileptic drugs used with lacosamide in patients with complete seizure control (group $A ; N=21$ )

\begin{tabular}{lc}
\hline Co-AED used with lacosamide & Patients $(\mathrm{n})^{\mathrm{a}}$ \\
\hline Levetiracetam & 9 \\
Valproate & $13[3]^{\mathrm{b}}$ \\
Lamotrigine & 3 \\
Zonisamide & 3 \\
Ethosuximide & $2[1]^{\mathrm{b}}$ \\
Clobazam & 2 \\
Topiramate & 1 \\
Rufinamide & 1 \\
Chlorazepate & 1 \\
Oxcarbazepine & 1 \\
\hline a 21 patients had complete control of seizures, but 3 discontinued \\
the treatment because of adverse effects. \\
b The numbers in brackets refer to patients in whom lacosamide \\
treatment was suspended. \\
co-AED = concomitant antiepileptic drug.
\end{tabular}


Table III. Etiology and types of seizure in patients with complete seizure control (group $A ; N=21$ )

\begin{tabular}{lll}
\hline Etiology & Type of seizure & Patients (n) \\
\hline Idiopathic & $\mathrm{S}+\mathrm{C}$ & 1 \\
& $\mathrm{C}$ & 1 \\
Symptomatic & $\mathrm{S}+\mathrm{C}+2^{\mathrm{a}} \mathrm{G}$ & 1 \\
& $\mathrm{~S}$ & 1 \\
& $\mathrm{C}$ & 3 \\
Presumably symptomatic & $\mathrm{C}+2^{\mathrm{a}} \mathrm{G}$ & 7 \\
& $\mathrm{~S}+\mathrm{C}$ & 1 \\
& $\mathrm{G}$ & 1 \\
& $\mathrm{C}$ & 3 \\
& $\mathrm{C}+2^{\mathrm{a}} \mathrm{G}$ & 2
\end{tabular}

$\mathbf{2}^{\mathrm{a}} \mathbf{G}=$ secondarily generalized seizure; $\mathbf{C}=$ complex focal seizure $\mathbf{G}=$ generalized seizure; $\mathbf{S}=$ simple focal seizure.

immune disease [Rasmussen's syndrome] (2.4\%). A high percentage of patients $(81.5 \%)$ had cognitive problems, of whom $56(43 \%)$ had serious retardation. The epileptic syndrome was identified in 26 cases, which included West syndrome (eight cases); Dravet syndrome (six cases); continuous spike-wave during slow sleep syndrome [CSWS] (five cases); Lennox syndrome, autosomal dominant nocturnal frontal lobe epilepsy, or Rasmussen's syndrome (two cases each); and Dulac devastating epilepsy (one case).

Lacosamide therapy was primarily used as an oral solution $(70.7 \%)$ or as a tablet; lacosamide was also initiated parenterally in three patients. The initial dose ranged from 1 to $2 \mathrm{mg} / \mathrm{kg} /$ day in the majority of cases $(89.2 \%)$, with the final dose being reached by 4 weeks in $45.3 \%$ of patients and by 12 weeks in $33.7 \%$ of patients; $20.7 \%$ reached the final dose in less than 4 weeks. The final mean dose was $6.80 \pm 2.39 \mathrm{mg} / \mathrm{kg} /$ day. Co-AEDs used in conjunction with lacosamide during the study included valproate (45.4\% of patients), levetiracetam $(39.2 \%)$, zonisamide $(17.7 \%)$, oxcarbazepine $(13.8 \%)$, clobazam $(13.8 \%)$, and topiramate (13.1\%).

\section{Efficacy Outcomes}

A total of 86 patients responded to lacosamide therapy $(66.2 \%)$, although five patients were not classified as responders, because of poor tolerability that resulted in lacosamide withdrawal.
Therefore, a total of 81 responders $(62.3 \%)$ were identified who made up the first three groups from the five categories, on the basis of their level of response to lacosamide therapy.

Group A: A total of 21 patients (16.2\%) had complete control of seizures (seizure suppression), although three patients experienced adverse effects that impeded the continuation of treatment. Therefore, complete control was observed in 18 patients $(13.8 \%)$, in whom a mean lacosamide dose of $6.97 \pm 2.15 \mathrm{mg} / \mathrm{kg} /$ day (range $4.61-13 \mathrm{mg} / \mathrm{kg} /$ day) was used. Among patients receiving mono- or bi-/polytherapy, levetiracetam ( 9 out of 18 cases; $50 \%$ ) and valproate (10 out of 18 cases; $55.5 \%$ ) were the two most commonly used co-AEDs in this group (table II). Etiology and types of seizure in group A are listed in table III; in the symptomatic group, one case of mitochondrial disease and three cases of MCD were reported.

Group B: Overall, 33 patients (25.4\%) achieved a $>75 \%$ reduction in seizure frequency, although poor tolerability led to drug withdrawal in two of these patients. Consequently, 31 patients (23.8\%) maintained this response level at a mean lacosamide dose of $6.40 \pm 2.48 \mathrm{mg} / \mathrm{kg} /$ day (range $2.14-13 \mathrm{mg} / \mathrm{kg} /$ day). Among patients receiving mono- or bi-/polytherapy, lacosamide was used concomitantly with levetiracetam in 11 patients $(32.3 \%)$ and with valproate in 14 patients (43.7\%)

Table IV. Concomitant antiepileptic drugs used with lacosamide in patients with seizure frequency control of $>75 \%$ (group $B ; n=33$ )

\begin{tabular}{ll}
\hline Co-AED used with lacosamide & Patients (n) \\
\hline Levetiracetam & $12[1]^{\mathrm{a}}$ \\
Valproate & 14 \\
Zonisamide & 5 \\
Topiramate & 2 \\
Oxcarbazepine & 8 \\
Carbamazepine & 1 \\
Clobazam & 6 \\
Rufinamide & 2 \\
Lamotrigine & 1 \\
Vigabatrin & 1 \\
Phenobarbital & 1 \\
\hline a The number in brackets refers to a case in whom lacosamide \\
$\quad$ treatment was suspended. \\
co-AED=concomitant antiepileptic drug.
\end{tabular}


Table V. Etiology and types of seizure in patients with seizure frequency control of $>75 \%$ (group $\mathrm{B} ; \mathrm{N}=33$ )

\begin{tabular}{llc}
\hline Etiology & Type of seizure & Patients $(\mathrm{n})$ \\
\hline Idiopathic & $\mathrm{S}+\mathrm{C}$ & 1 \\
Symptomatic & $\mathrm{S}$ & 1 \\
& $\mathrm{C}$ & 3 \\
& $\mathrm{C}+2^{\mathrm{a}} \mathrm{G}$ & 13 \\
Presumably symptomatic & $\mathrm{S}+\mathrm{C}+2^{\mathrm{a}} \mathrm{G}$ & 5 \\
& $\mathrm{C}$ & 7 \\
& $\mathrm{~S}+\mathrm{C}$ & 2 \\
& $\mathrm{~S}+\mathrm{C}+2^{\mathrm{a}} \mathrm{G}$ & 1 \\
\hline
\end{tabular}

$\mathbf{2}^{\mathbf{a}} \mathbf{G}=$ secondarily generalized seizure; $\mathbf{C}=$ complex focal seizure $\mathbf{S}=$ simple focal seizure.

[table IV]. Etiology and types of seizure in group $\mathrm{B}$ are listed in table $\mathrm{V}$; in the symptomatic group, five cases of MCD were observed, but no cases of mitochondrial disease were reported.

Group C: A seizure frequency reduction of $>50 \%$ to $75 \%$ was seen in 32 patients $(24.6 \%)$, with a mean lacosamide dose of $6.63 \pm 2.33 \mathrm{mg} / \mathrm{kg} / \mathrm{day}$ (range $2.4-14.3 \mathrm{mg} / \mathrm{kg} /$ day). Among patients receiving mono- or bi-/polytherapy, lacosamide was used concomitantly with levetiracetam in 13 patients $(40.6 \%)$, with valproate in 13 patients $(40.6 \%)$, and with zonisamide in seven patients $(21.8 \%)$ [table VI]. Etiology and types of seizure in group $\mathrm{C}$ are listed in table VII; in the symptomatic group, three cases of mitochondrial disease and four cases of MCD were observed.

Group D: No change in seizure frequency was observed in 39 patients (30\%), who received an average dose of $7.26 \pm 2.62 \mathrm{mg} / \mathrm{kg} /$ day (range $5-20 \mathrm{mg} / \mathrm{kg} / \mathrm{day})$. The co-AEDs that were used most often in groups A, B, and C were used less frequently in group $\mathrm{D}$. Among patients receiving mono- or bi-/polytherapy, lacosamide was used concomitantly with levetiracetam in 16 patients (41\%), with valproate in 21 patients $(53.8 \%)$, and with topiramate in 12 patients $(30.8 \%)$ [table VIII]. Etiology and types of seizure in group D are listed in table IX; in the symptomatic group, mitochondrial disease and MCD were observed in one and four cases, respectively.

Group E: An increase in seizure frequency was seen in five patients $(3.8 \%)$. The mean lacosamide dose in this group was $6.16 \pm 0.52 \mathrm{mg} / \mathrm{kg} /$ day (range $5.6-7 \mathrm{mg} / \mathrm{kg} /$ day). Lacosamide was not used con- comitantly with levetiracetam or valproate in these patients, and no patients were receiving three or more co-AEDs (table X). Etiology and types of seizure in group $\mathrm{E}$ are listed in table $\mathrm{XI}$; in the symptomatic group, one case of MCD was reported.

Figure 1 shows the pattern of the treatment response in this population of children with refractory epilepsy. No statistically significant differences in the mean lacosamide doses were seen between the different groups ( $p=0.499$; Kruskal-Wallis test). However, the mean lacosamide doses tended to be similar in groups $\mathrm{A}, \mathrm{B}$, and $\mathrm{C}$, but higher in group $\mathrm{D}$, with the aim of increasing the therapeutic response.

No relationship between the response to lacosamide therapy and epileptic syndrome was observed. Two patients with Lennox-Gastaut syndrome reported a focal seizure reduction of $>50 \%$. One patient with continuous partial epilepsy (Rasmussen's syndrome) appeared to achieve control of seizures with lacosamide therapy.

\section{Safety and Tolerability (Unfavorable and Favorable Secondary Effects)}

Adverse effects were reported by patients and their families in 39 cases $(30 \%)$ following treatment with lacosamide. In 16 of these cases, the

Table VI. Concomitant antiepileptic drugs used with lacosamide in patients with seizure frequency control of $>50 \%$ (group $\mathrm{C} ; \mathrm{N}=32$ )

\begin{tabular}{lc}
\hline Co-AED used with lacosamide & Patients $(\mathrm{n})$ \\
\hline Levetiracetam & 13 \\
Oxcarbazepine & 3 \\
Chlorazepate & 4 \\
Valproate & 13 \\
Zonisamide & 7 \\
Topiramate & 3 \\
Lamotrigine & 5 \\
Carbamazepine & 3 \\
Rufinamide & 2 \\
Phenobarbital & 2 \\
Clobazam & 2 \\
Phenytoin/diphenylhydantoin & 4 \\
Tiagabine & 1 \\
Vigabatrin & 1 \\
Vagal stimulator & 1 \\
\hline co-AED = concomitant antiepileptic drug. &
\end{tabular}


Table VII. Etiology and types of seizure in patients with seizure frequency control of $>50 \%$ (group $\mathrm{C} ; \mathrm{N}=32$ )

\begin{tabular}{lll}
\hline Etiology & Type of seizure & Patients (n) \\
\hline Idiopathic & $\mathrm{S}+\mathrm{C}$ & 1 \\
Symptomatic & $\mathrm{S}$ & 1 \\
& $\mathrm{C}$ & 5 \\
& $\mathrm{~S}+\mathrm{C}$ & 2 \\
& $\mathrm{~S}+2^{\mathrm{a}} \mathrm{G}$ & 1 \\
& $\mathrm{C}+2^{\mathrm{a}} \mathrm{G}$ & 9 \\
Presumably symptomatic & $\mathrm{S}+\mathrm{C}+2^{\mathrm{a}} \mathrm{G}$ & 3 \\
& $\mathrm{C}$ & 3 \\
& $\mathrm{~S}+\mathrm{C}$ & 1 \\
& $\mathrm{C}+2^{\mathrm{a}} \mathrm{G}$ & 7
\end{tabular}

$\mathbf{2}^{\mathbf{a}} \mathbf{G}=$ secondarily generalized seizure; $\mathbf{C}=$ complex focal seizure $\mathbf{S}=$ simple focal seizure.

effects were initial and transient; in four cases, the effects were tolerated without requiring dose modification; in six cases, the effects disappeared or were tolerated by lowering the lacosamide dose; and in 13 cases, the effects required cessation of lacosamide. The mean dose of lacosamide in the 39 patients who experienced an adverse effect was $7.11 \pm 3.10 \mathrm{mg} / \mathrm{kg} / \mathrm{day}$, compared with $6.56 \pm 2.21 \mathrm{mg} / \mathrm{kg} / \mathrm{day}$ in the 91 patients who did not experience any adverse effects; no statistically significant difference was seen between these two doses ( $\mathrm{p}=0.304$; Mann-Whitney test).

No cardiovascular effects were observed in our patients. There were also no alterations in conventional laboratory tests (complete blood count, transaminasemia, amylasemia, blood glucose, creatininemia, cholesterolemia, and triglyceridemia), and no significant changes in EEG records.

The most prevalent adverse effects were nausea and vomiting (13 cases), instability (ten cases), dizziness (five cases), nystagmus (three cases), somnolence (three cases), weakness (two cases), and adynamia (two cases). Anorexia, disorientation, asthenia, headache, insomnia, irritability, attention deficit, agitation, drop in academic achievement, psychotic reaction, vision impairment, neck stiffness, tonic upgaze, sialorrhea, and focal epileptic status were much less common effects (one case each).

In ten patients, striking symptoms were observed, including instability, difficulty walking, an inability to relate subjective elements, and blurred vision or dizziness. In five cases, symptom intensity remained unchanged, despite an immediate dose decrease, which eventually led to discontinuation of treatment. In all cases, symptoms peaked with the $\mathrm{C}_{\max }$ occurring between 2 and 5 hours after drug administration, with no direct relationship to the dose, speed of dose adjustment, or use of coAEDs. Adverse effects resulting in discontinuation of lacosamide are detailed in table XII.

A significant improvement in behavior and the speed of response to stimuli was reported by the parents of 17 patients $(13.0 \%)$ in groups A and $\mathrm{B}$, which may have been related to the use of lacosamide.

\section{Discussion}

The results of this open-label study suggest that lacosamide therapy may be an effective treatment option in children with refractory epilepsy. The epilepsy diagnosis criteria used in our study were the same as those used in two previous studies evaluating lacosamide therapy in children. ${ }^{[9,10]}$ In our study, a much larger sample of patients was enrolled and a more favorable response was observed, compared with the studies conducted by Gavatha et al. ${ }^{[10]}$ and Chez et al. ${ }^{[9]}$

Table VIII. Concomitant antiepileptic drugs used with lacosamide in patients with no change in seizure frequency (group $\mathrm{D} ; \mathrm{N}=39$ )

\begin{tabular}{lc}
\hline Co-AED used with lacosamide & Patients (n) \\
\hline None & 1 \\
Levetiracetam & 16 \\
Valproate & 21 \\
Zonisamide & 8 \\
Clobazam & 8 \\
Topiramate & 12 \\
Oxcarbazepine & 5 \\
Phenobarbital & 3 \\
Lamotrigine & 4 \\
Carbamazepine & 1 \\
Primidone & 4 \\
Rufinamide & 1 \\
Ethosuximide & 1 \\
Pregabalin & 1 \\
Chlorazepate & 1 \\
Phenytoin & 1 \\
\hline co-AED = concomitant antiepileptic drug. &
\end{tabular}


Table IX. Etiology and types of seizure in patients with no change in seizure frequency (group $\mathrm{D} ; \mathrm{N}=39$ )

\begin{tabular}{lll}
\hline Etiology & Type of seizure & Patients $(\mathrm{n})$ \\
\hline Idiopathic & $\mathrm{C}$ & 1 \\
& $\mathrm{~S}+2^{\mathrm{a}} \mathrm{G}$ & 1 \\
Symptomatic & $2^{\mathrm{a}} \mathrm{G}$ & 1 \\
& $\mathrm{C}$ & 3 \\
& $\mathrm{~S}$ & 1 \\
& $\mathrm{~S}+\mathrm{C}$ & 1 \\
& $\mathrm{~S}+2^{\mathrm{a}} \mathrm{G}$ & 2 \\
& $\mathrm{C}+2^{\mathrm{a}} \mathrm{G}$ & 7 \\
& $\mathrm{~S}+\mathrm{C}+2^{\mathrm{a}} \mathrm{G}$ & 6 \\
Presumably symptomatic & $2^{\mathrm{a}} \mathrm{G}$ & 8 \\
& $\mathrm{C}$ & 2 \\
& $2^{\mathrm{a}} \mathrm{G}$ & 3 \\
& $\mathrm{C}+2^{\mathrm{a}} \mathrm{G}$ & 3 \\
\hline
\end{tabular}

$\mathbf{2}^{\mathbf{a}} \mathbf{G}=$ secondarily generalized seizure; $\mathbf{C}=$ complex focal seizure; $\mathbf{S}=$ simple focal seizure.

We reported seizure suppression in $16.2 \%$ of patients, compared with $11.1 \%$ in the study conducted by Gavatha et al..$^{[10]}$ and $4.3 \%$ in the study conducted by Chez et al. ${ }^{[9]}$ The favorable response in our study may have been a reflection of the higher lacosamide doses that were used (a mean dose $6.8 \mathrm{mg} / \mathrm{kg} / \mathrm{day}$ ), compared with those used by Gavatha et al. ${ }^{[10]}(5.17 \mathrm{mg} / \mathrm{kg} / \mathrm{day})$ and Chez et al. ${ }^{[9]}$ (3.6 mg/kg/day).

Our results are suggestive of greater efficacy with the combination of lacosamide and an AED with a complementary mechanism of action, such as levetiracetam (which binds to synaptic vesicle proteins) or valproate (which is a GABAergic enhancer and has activity at the sodium channel). ${ }^{[12]}$ Conversely, the combination of lacosamide with various agents that act on sodium channels (e.g. benzodiazepine, carbamazepine, ethosuximide, lamotrigine, oxcarbazepine, phenytoin, phenobarbital, topiramate, or zonisamide) appeared to be less efficacious in this population. Moreover, it has been suggested that the association of lacosamide with other sodium channel-acting AEDs can induce neurotoxicity. ${ }^{[12]}$ Interestingly, the proportion of patients who used co-AEDs was greater in groups A and B (i.e. patients with a favorable response to lacosamide therapy), although it should be noted that this study was not powered to make such comparisons.
We did not observe any relationship between the response to lacosamide therapy and epileptic syndrome. However, two patients with LennoxGastaut syndrome reported a focal seizure reduction of $>50 \%$, which is in contrast to the worsening of seizure control that has been previously reported. ${ }^{[13]}$ Moreover, we achieved great success in one of the patients with continuous partial epilepsy (Rasmussen's syndrome), whose seizures appeared to be controlled by lacosamide therapy. Indeed, a similar outcome was observed in a 72-year-old patient with refractory partial epileptic status secondary to an ischemic lesion. ${ }^{[14]}$

Although the results of this study are encouraging and of great interest, the study had limitations inherent to its design. The open-label design of the study allowed for the potential that the results might be affected by bias. The relatively small number of patients limited the study power, although this was a consequence of the 12-month recruitment period. Another limitation of the current study was the mixed patient population. Patients with a variety of medication-resistant seizures were enrolled in the trial, including those with symptomatic generalized epilepsy syndromes and those with partial epilepsies. Because of the variety of underlying etiologies in this population, the results may not be generalizable across all types of pediatric patients.

Lacosamide appeared to be generally well tolerated in this population. We did not observe a dose-dependent relationship between lacosamide therapy and the development of adverse effects. Indeed, the patient who received the highest lacosamide dose $(20 \mathrm{mg} / \mathrm{kg} /$ day $)$ did not experience any

Table X. Concomitant antiepileptic drugs used with lacosamide in patients with an increase in seizure frequency (group $\mathrm{E} ; \mathrm{N}=5$ )

\begin{tabular}{ll}
\hline Co-AED used with lacosamide & Patients $(\mathrm{n})$ \\
\hline Oxcarbazepine & 2 \\
Valproate & 3 \\
Topiramate & 2 \\
Zonisamide & 2 \\
Lamotrigine & 2 \\
Vigabatrin & 1 \\
Chlorazepate & 1 \\
\hline co-AED = concomitant antiepileptic drug. &
\end{tabular}


Table XI. Etiology and types of seizure in patients with an increase in seizure frequency (group $\mathrm{E} ; \mathrm{N}=5$ )

\begin{tabular}{lll}
\hline Etiology & Type of seizure & Patients $(\mathrm{n})$ \\
\hline Symptomatic & $\mathrm{S}+\mathrm{C}$ & 1 \\
& $\mathrm{~S}+2^{\mathrm{a}} \mathrm{G}$ & 1 \\
& $\mathrm{C}+2^{\mathrm{a}} \mathrm{G}$ & 1 \\
Presumably symptomatic & $\mathrm{S}$ & 1 \\
& $2^{\mathrm{a} G}$ & 1 \\
\hline
\end{tabular}

$\mathbf{2}^{\mathbf{a}} \mathbf{G}=$ secondarily generalized seizure; $\mathbf{C}=$ complex focal seizure $\mathbf{S}=$ simple focal seizure.

adverse effects. Moreover, a very large dose of lacosamide, used in a suicide attempt, did not result in death or permanent injury; complete physical recovery was achieved after several days. ${ }^{[15]}$ Plasma drug levels were not determined in our study, although determination of saliva drug concentrations is a new alternative that may provide a more objective method of analysis in the near future. ${ }^{[16]}$ As a consequence, this may enable a more rational method of adjusting lacosamide doses.

The literature suggests that adverse effects associated with lacosamide therapy are generally mild-to-moderate in severity at doses of up to
$600 \mathrm{mg} /$ day. ${ }^{[3,4,6]}$ Although adverse effects were observed in $30 \%$ of patients in our study, these effects led to drug withdrawal in only $10 \%$ of the overall study population. Additionally, the series by Gavatha et al. ${ }^{[10]}$ reported a similar incidence of adverse effects (33\%). In the study by Chez et al., ${ }^{[9]}$ adverse effects were observed in $8.6 \%$ of cases, which is a slightly lower rate, but lower doses were also used. However, there continues to be doubt concerning the hypothetical relationship between adverse effects and dose, which we were unable to confirm either way.

The marked instability, difficulty walking, and blurred vision that were observed here in ten patients have also been reported previously in a series of adult patients. ${ }^{[17]}$ In five of our cases, symptom intensity remained unchanged, despite an immediate dose decrease, which eventually led to suspension of treatment. Furthermore, these symptoms differed significantly between patients, which prevented determination of a convincing pathophysiological explanation, or the relationship between these symptoms and the use of other AEDs. Further investigation of these effects

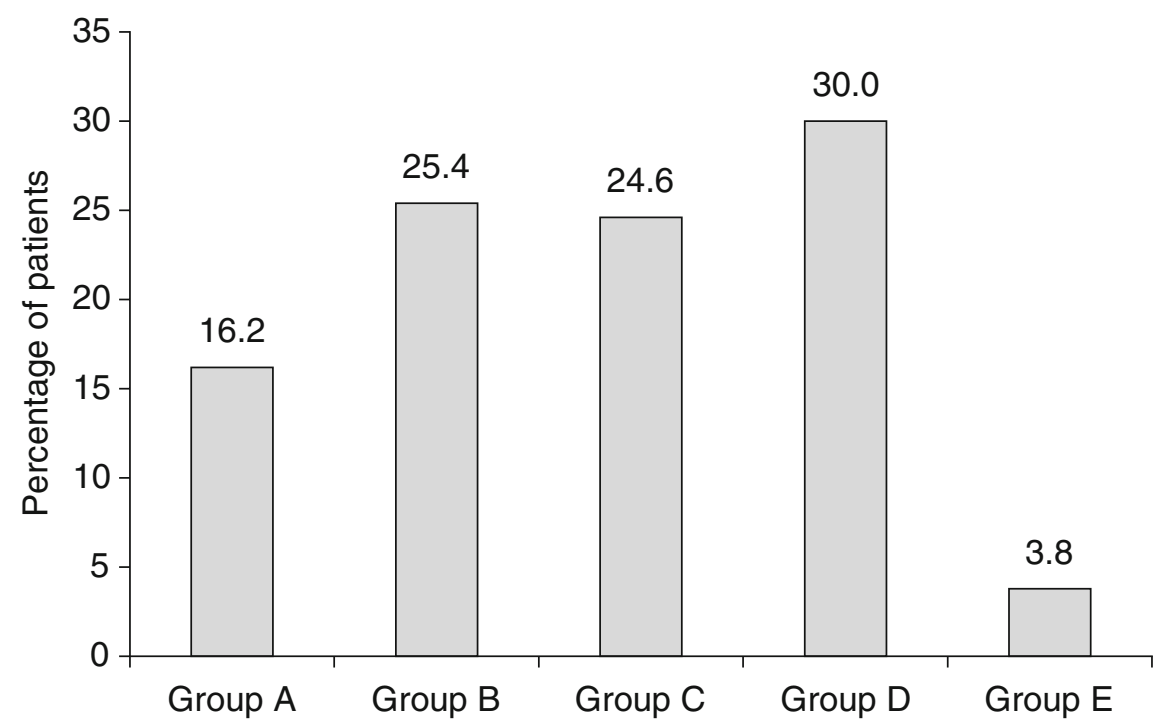

Fig. 1. Pattern of the treatment response (change in seizure frequency) to lacosamide therapy in children aged $<16$ years with refractory epilepsy: Group A, seizure suppression; group B, $>75 \%$ reduction in seizure frequency; group C, $>50 \%$ to $75 \%$ reduction in seizure frequency; epilepsy: Group A, seizure suppression; group B, $>75 \%$ reduction in seizure frequency; group $C,>50 \%$ to $75 \%$ reduction in seizure frequency;
group D, no change in seizure frequency; group $\mathrm{E}$, increase in seizure frequency. The mean \pm standard deviation lacosamide doses $(\mathrm{mg} / \mathrm{kg} /$ day) were: group A, $6.97 \pm 2.15 \mathrm{mg} / \mathrm{kg} /$ day; group B, $6.40 \pm 2.48 \mathrm{mg} / \mathrm{kg} /$ day; group C, $6.63 \pm 2.33 \mathrm{mg} / \mathrm{kg} /$ day; group D, $7.26 \pm 2.62$ $\mathrm{mg} / \mathrm{kg} / \mathrm{day} ;$ group E, $6.16 \pm 0.52 \mathrm{mg} / \mathrm{kg} /$ day. 
Table XII. Reasons for discontinuation of lacosamide $(\mathrm{N}=13)$

\begin{tabular}{ll}
\hline Reason for discontinuation & Patients $(\mathrm{n})^{\mathrm{a}}$ \\
\hline Adverse effects & 10 \\
Instability & 5 \\
Nausea/vomiting & 3 \\
Dizziness & 1 \\
Anorexia & 1 \\
Disorientation & 1 \\
Drowsiness & 1 \\
Psychotic reaction & 1 \\
Stiff neck & 1 \\
Visual disorder & 1 \\
Agitation & 1 \\
Status epilepticus & 1 \\
Therapeutic inefficacy with no adverse effects & 3 \\
\hline Some patients experienced more than one adverse effect.
\end{tabular}

a Some patients experienced more than one adverse effect.

is required in randomized, controlled trials to fully elucidate any causal factors in this patient population.

No cardiovascular effects were observed in our patients. In contrast, lacosamide has been associated with atrial flutter/atrial fibrillation at doses of $600 \mathrm{mg}$ /day or above in adults with epilepsy. ${ }^{[5]}$ Furthermore, we did not observe any alterations in conventional laboratory tests or significant changes in EEG records. However, we did not have the opportunity to assess favorable effects of lacosamide on photoparoxysmal responses, which have recently been reported. ${ }^{[18]}$

\section{Conclusion}

In summary, lacosamide appears to be an effective and generally well tolerated AED in children and adolescents with pharmaco-resistant focal epileptic seizures. However, the instability, accompanied by difficulty walking and blurred vision, that was observed in ten patients requires further investigation. On the basis of these results, we recommend an initial dose of $1-2 \mathrm{mg} / \mathrm{kg} /$ day in children, followed by gradual uptitration every 4-7 days, according to tolerability, until a mean dose of $6-9 \mathrm{mg} / \mathrm{kg} /$ day is achieved; the drug can be administered twice daily (i.e. once every 12 hours). Although care must be taken with concomitant use of AEDs that act on sodium channels, adjunctive therapy with lacosamide (a non-traditional sodiumchannel blocking AED) significantly reduced seizure frequency regardless of co-administration of traditional sodium-channel blockers in this open-label trial. ${ }^{[19]}$ Randomized controlled trials of lacosamide are needed to confirm and validate the efficacy and safety results observed here in this pediatric population.

\section{Acknowledgments}

This study was funded by Dr. Carlos Casas-Fernández. Medical writing and journal styling assistance was provided by Maxwell Chang and Lucy Whitehouse, and postsubmission writing assistance was provided by Tracy Harrison, all of inScience Communications, Springer Healthcare; this assistance was funded by Dr. Carlos Casas-Fernández, The authors have no conflicts of interest to declare.

The authors confirm that they have read the Journal's position on issues involved in ethical publication and affirm that this report is consistent with those guidelines.

\section{Appendix: Lacosamide Spanish Study Group Members}

Dr. Alarcón-Martínez (Hospital Universitario Virgen de la Arrixaca, Murcia); Dr. ArrabalFernández (Hospital Universitario Virgen de las Nieves, Granada); Dr. Cabrera-López (Hospital Universitario Materno-Infantil, Las Palmas de Gran Canaria, Canary Islands): Dr. CaminoLeón (Hospital Universitario Reina Sofía, Cordoba); Dr. Campistol-Plana (Hospital Universitario San Juan de Dios, Barcelona); Dr. Campos-Castello (Hospital Clínico San Carlos, Madrid); Dr. Casas-Fernández (Hospital Universitario Virgen de la Arrixaca, Murcia); $D r$. Domingo Jiménez (Hospital Universitario Virgen de la Arrixaca, Murcia); Dr. Duque-Fernández (Hospital Universitario Virgen de La Candelaria, Santa Cruz de Tenerife); Dr. Eiris-Puñal (Hospital Clínico Universitario, Santiago de Compostela); Dr. García-Peñas (Hospital Universitario Marqués de Valdecilla, Santander); Dr. HerranzFernández (Universidad de Cantabria, Santander); Dr. Ibáñez-Micó (Hospital Universitario Virgen de la Arrixaca, Murcia); Dr. Jover-Cerda (Hospital General de Elda, Alicante); Dr. Lara- 
Herguedas (Hospital Universitario Puerta de Hierro-Majadahonda, Madrid); Dr. LópezLafuente (Hospital San Pedro de Alcántara, Cáceres); Dr. Madruga-Garrido (Hospital Universitario Virgen del Rocío, Seville); Dr. MartínezBermejo (Hospital Universitario La Paz, Madrid); Dr. Martínez-Salcedo (Hospital Universitario Virgen de la Arrixaca, Murcia); Dr. Puche-Mira (Hospital Universitario Virgen de la Arrixaca, Murcia); Dr. Roldán-Aparicio (Hospital Universitario Virgen de las Nieves, Granada); Dr. Rufo-Campos (Instituto Hispalense de Pediatría, Seville); Dr. Santos-Borbujo (Hospital Clínico Universitario, Salamanca); Dr. SmeyersDurá (Hospital Universitario La Fe, Valencia); Dr. Teva-Galán (Hospital General de Elda, Alicante); Dr. Valera-Párraga (Hospital Universitario Virgen de la Arrixaca, Murcia).

\section{References}

1. Bolin K, Berggren F, Forsgren L. Lacosamide as treatment of epileptic seizures: cost utility results for Sweden. Acta Neurol Scand 2010 Jun; 121 (6): 406-12

2. Chu-Shore CJ, Thiele EA. New drugs for paediatric epilepsy. Semin Pediatr Neurol 2010 Dec; 17 (4): 214-23

3. Chung SS. New treatment option for partial-onset seizures: efficacy and safety of lacosamide. Ther Adv Neurol Disord 2010 Mar; 3 (2): 77-83

4. Kelemen A, Halasz P. Lacosamide for the prevention of partial onset seizures in epileptic adults. Neuropsychiatr Dis Treat 2010; 6: 465-71

5. Chung SS. Atrial flutter/atrial fibrillation associated with lacosamide for partial seizures. Epilepsy Behav 2010; 18 (3): $322-4$

6. Chung SS. Lacosamide: new adjunctive treatment option for partial-onset seizures. Expert Opin Pharmacother 2010 Jun; 11 (9): 1595-602

7. Ben-Menachem E, Biton V, Jatuzis D, et al. Efficacy and safety of oral lacosamide as adjunctive therapy in adults with partial-onset seizures. Epilepsia 2007 Jul; 48 (7): 1308-17

8. Halasz P, Kalviainen R, Mazurkiewicz-Beldzinska M, et al. Adjunctive lacosamide for partial-onset seizures: efficacy and safety results from a randomized controlled trial. Epilepsia 2009 Mar; 50 (3): 443-53

9. Chez MG, Sacramento CA. Lacosamide as add-on therapy in paediatric epilepsy: retrospective clinical experience [abstract]. Neurology 2010; 74 Suppl. 2: 74

10. Gavatha M, Ioannou I, Papavasiliou AS. Efficacy and tolerability of oral lacosamide as adjunctive therapy in paediatric patients with pharmacoresistant focal epilepsy. Epilepsy Behav 2010 Apr; 20 (51 Suppl. 4): 691-3

11. Kwan P, Arzimanoglou A, Berg AT, et al. Definition of drug resistant epilepsy: consensus proposal by the ad hoc Task Force of the ILAE Commission on Therapeutic Strategies. Epilepsia 2010 Jun; 51 (6): 1069-77

12. Novy J, Patsalos PN, Sander JW, et al. Lacosamide neurotoxicity associated with concomitant use of sodium channelblocking antiepileptic drugs: a pharmacodynamic interaction? Epilepsy Behav 2011 Jan; 20 (1): 20-3

13. Cuzzola A, Ferlazzo E, Italiano D, et al. Does lacosamide aggravate Lennox-Gastaut syndrome? Report on three consecutive cases. Epilepsy Behav 2010 Dec; 19 (4): 650-1

14. Turpin-Fenoll L, Millan-Pascual J, Navarro-Munoz S, et al. The use of oral lacosamide in a patient with refractory partial epileptic status. Rev Neurol 2010 May 16; 50 (10): 603-6

15. Bauer S, David Rudd G, Mylius V, et al. Lacosamide intoxication in attempted suicide. Epilepsy Behav 2010 Apr; 17 (4): $549-51$

16. Greenaway C, Ratnaraj N, Sander JW, et al. Saliva and serum lacosamide concentrations in patients with epilepsy. Epilepsia 2011 Feb; 52 (2): 258-63

17. Wehner T, Bauer S, Hamer HM, et al. Six months of postmarketing experience with adjunctive lacosamide in patients with pharmacoresistant focal epilepsy at a tertiary epilepsy center in Germany. Epilepsy Behav 2009 Nov; 16 (3): 423-5

18. Parkerson KA, Reinsberger C, Chou SH, et al. Lacosamide in the treatment of acute recurrent seizures and periodic epileptiform patterns in critically ill patients. Epilepsy Behav 2011 Jan; 20 (1): 48-51

19. Sake JK, Hebert D, Isojarvi J, et al. A pooled analysis of lacosamide clinical trial data grouped by mechanism of action of concomitant antiepileptic drugs. CNS Drugs 2010 Dec 1; 24 (12): 1055-68

Correspondence: Dr. Carlos Casas-Fernández, Neuropediatric Department, Hospital Universitario Virgen de la Arrixaca, 30120-El Palmar, Murcia, Spain.

E-mail: casascarlos@telefonica.net 